\title{
TERAPÊUTICA MEDICAMENTOSA NA ISQUEMIA E REPERFUSÃO MESENTÉRICA: REVISÃO DA LITERATURA
}

\author{
CARLOS HENRIQUE MARQUES DOS SANTOS ${ }^{1}$, JOSÉ CARLOS DORSA VIEIRA PONTES ${ }^{2}$, \\ OTONI MOREIRA GOMES ${ }^{3}$
}

\author{
${ }^{1}$ Mestre em Medicina (FCVSFA) - Membro Titular da Sociedade Brasileira de Coloproctologia, ${ }^{2}$ Prof Adjunto de \\ Cirurgia Cardiovascular - UFMS, ${ }^{3}$ Prof Titular de Clínica Cirúrgica da UFMG
}

SANTOS CHM, PONTES JCDV, GOMES OM. Terapêutica Medicamentosa na Isquemia e Reperfusão Mesentérica: Revisão da Literatura. Rev bras Coloproct, 2006; 26(1):28-33.

RESUMO: Até pouco tempo atrás se acreditava que na isquemia mesentérica todas as alterações orgânicas desta afecção eram devidas à obstrução total ou parcial do fluxo arterial intestinal. Recentes descobertas quanto à fisiopatologia do processo de isquemia e reperfusão mesentérica demonstraram que os radicais livres, principalmente, atuam durante a reperfusão, levando à lesão tecidual muito mais importante do que as lesões que ocorrem na fase de isquemia isoladamente. Assim, surge uma nova possibilidade terapêutica além do tratamento cirúrgico, em que uma determinada substância poderia atuar de modo a inibir ou minimizar a cascata de alterações no nível celular que culminam na lesão e morte celular. Realizamos uma ampla revisão da literatura médica atual pelos bancos de dados LILACS e MEDLINE, visando verificar quais os fármacos estudados para este fim e os resultados obtidos nas pesquisas. Constatamos que inúmeras substâncias têm sido avaliadas em estudos experimentais, em sua maioria utilizando ratos e a maioria não apresentou resultados satisfatórios a ponto de permitirem seu emprego na prática clínica; algumas, entretanto, apresentaram resultados promissores, necessitando ainda de novos estudos a fim de se descobrir uma substância que possa ser empregada em seres humanos em situações de isquemia e reperfusão mesentérica, a fim de se evitar tratamentos intervencionistas com altos índices de morbi-mortalidade.

Descritores: Isquemia, reperfusão, cirurgia experimental

\section{INTRODUÇÃO}

A isquemia é uma condição de interrupção no suprimento de oxigênio e nutrimentos para uma determinada área, durante um período, devido a uma deficiência de fornecimento de sangue, o que pode acarretar a morte tecidual ${ }^{1}$. Recentemente, surgiram evidências de que a lesão dos tecidos não estaria somente limitada à isquemia, podendo se estender ou agravar com a reperfusão ${ }^{2,3}$.

PARKS \& GRANGER ${ }^{4}$ demonstraram que três horas de isquemia seguidas por uma hora de reperfusão determinavam maior lesão na mucosa intestinal do que quatro horas de exclusiva isquemia.

Em relação à isquemia intestinal, não faz muito tempo, o tratamento restringia-se a ressecções

Trabalho realizado no Hospital Regional de Mato Grosso do Sul.

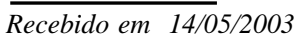

Aceito para publicação em 14/03/2006 
intestinais associadas ou não a embolectomias 5, 6, 7, 8, 9, 10 . Tais procedimentos continuam sendo justificáveis em situações nas quais não mais é possível realizar a reperfusão, como na gangrena intestinal, por ser esta lesão irreversível ${ }^{10}$. Entretanto, em muitas situações há lugar para um tratamento que bloqueie a seqüência de eventos do processo de isquemia e reperfusão, de modo a inibir ou minimizar seus efeitos danosos aos tecidos ${ }^{11,12,13}$.

Há também situações em que o tratamento operatório se impõe, porém, isoladamente, é insuficiente para a completa resolução da afecção, visto que a reperfusão mesentérica traz danos teciduais e sistêmicos que devem ser evitados.

A isquemia mesentérica aguda consiste na ausência ou diminuição acentuada do fluxo sangüíneo por comprometimento arterial, venoso ou da microcirculação intestinal ${ }^{10}$.

Com o conhecimento da seqüência de eventos que levam à lesão tecidual decorrente principalmente da produção dos radicais livres, acredita-se que possam haver formas de atuar em algum nível da reação em cadeia do processo de isquemia e reperfusão, de modo a inibir a injúria tecidual.

Assim, inúmeras substâncias têm sido estudadas com o objetivo de evitar ou minimizar os efeitos maléficos da isquemia e reperfusão mesentérica em situações como as acima referidas.

Desta forma o objetivo deste trabalho é fazer uma revisão da literatura médica a fim de se conhecer o estado atual das pesquisas que visam encontrar substâncias capazes de prevenir ou minimizar as lesões de isquemia e reperfusão mesentérica.

\section{DISCUSSÃO}

SANTOS ${ }^{14}$ demonstrou recentemente que o propofol foi capaz de minimizar as lesões de isquemia e reperfusão mesentérica em ratos, em trabalho no qual submeteu os animais à isquemia durante 30 minutos por oclusão da artéria mesentérica cranial seguida de reperfusão por 60 minutos. Os animais do grupo propofol foram comparados aos do grupo controle que receberam solução salina por via intravenosa, obtendo-se médias de classificação histológica de 3,3 e 1,85 para os grupos controle e propofol, respectivamente, demonstrando uma diferença estatisticamente significativa entre os grupos. Utilizaram a classificação histológica de CHIU et al. ${ }^{15}$, descrita em 1970, que tornou-se clássica na avaliação de lesão tecidual intestinal por isquemia e reperfusão, sendo utilizada por diversos autores ${ }^{16-25}$ para esta finalidade. Esta classificação vai de grau zero, em que a mucosa é normal, até grau cinco, em que se observam as lesões mais severas com destruição total das vilosidades.

KAÇMAZ, OZTURK, KARAAYVAZ, GUVEN e DURAK ${ }^{22}$, estudando o Alopurinol como antioxidante na isquemia e reperfusão intestinal, obtiveram média 4,6 na classificação histológica de CHIU et al. ${ }^{15}$ para o grupo controle e 1,8 para o grupo Alopurinol. Estes autores utilizaram 5 ratos SpragueDawley em cada grupo e mantiveram os animais por quarenta minutos de isquemia seguidos por quarenta minutos de reperfusão. Apesar dos resultados positivos, o número de animais estudados foi bastante reduzido.

ONEN, DEVECI, INALOZ, ISIK e KILINC ${ }^{21}$ obtiveram média de classificação histológica 4,3 para o grupo controle e 2 para o grupo que recebeu extrato de Ginkgo-biloba, como agente anti-oxidante antes da laparotomia. Porém, apesar de terem deixado os ratos sob isquemia mesentérica por trinta minutos, o período de reperfusão foi de apenas vinte minutos. YONGMING, YAN, YE, ZHIGUO e ZHIYONG ${ }^{11}$ observaram que ratos submetidos a isquemia por oclusão da artéria mesentérica superior por quarenta e cinco minutos e posteriormente reperfundidos, apresentaram níveis de fator de necrose tumoral plasmáticos duas vezes maior após 2 horas de reperfusão em comparação ao grupo com trinta minutos. $\mathrm{O}$ fator de necrose tumoral foi utilizado para avaliar lesão tecidual pós-isquemia e reperfusão. Desta forma, é possível que com período de reperfusão maior os resultados de ONEN et al. ${ }^{21}$ não fossem tão bons.

BIONDO-SIMÕES et al. ${ }^{18}$ estudaram o uso de eritromicina administrada previamente à isquemia e reperfusão mesentérica em ratos e observaram não haver benefício utilizando tal substância em relação ao grupo controle.

Estudando a L-arginina como protetor da injúria tecidual na isquemia e reperfusão intestinal, WARD, LAWSON, GALLAGHER, CONNER e SHEA-DONOHUE ${ }^{20}$ obtiveram classificação 
histológica média de 3,9 para o grupo controle, 2,2 com a administração de L-arginina antes da isquemia e 3,1 antes da reperfusão, sugerindo haver potencial benefício de tal substância na prevenção da injúria tecidual. Também GUO, CHAN, FUNG, CHAN e TAM ${ }^{19}$ estudaram a L-arginina e observaram um aumento na produção de óxido nítrico e conseqüente redução da injúria pós-isquemia e reperfusão intestinal. De maneira oposta, KHANNA, ROSSMAN, FUNG e CATY ${ }^{26}$ afirmaram que o óxido nítrico apresenta fundamental participação na isquemia e reperfusão, estando o aumento de seus níveis relacionados a proporcional aumento na injúria tecidual. DURAKBASA, DAGLI, MOUNI et al. ${ }^{27}$ também afirmaram que os níveis elevados de óxido nítrico estão associados a injúria tecidual na isquemia e reperfusão. Com tal controvérsia, apesar de indícios de proteção tecidual oferecida pela L-arginina, fica difícil conhecer seu real mecanismo de ação, necessitando outros estudos mais aprofundados para o esclarecimento a respeito da participação do óxido nítrico na isquemia e reperfusão tecidual.

LEE, McCAULEY, KONG e HALL ${ }^{16}$ não observaram diferença na classificação histológica entre o grupo controle e o grupo que recebeu glicina previamente à isquemia em seu estudo.

Em nosso meio, MACARENCO, TAKAHAGI, BARDELLA, SEQUEIRA e YOSHIDA 17 utilizaram também a classificação histológica de CHIU et al. ${ }^{15}$ na análise de seus resultados em trabalho comparando a ação do extrato de Ginkgo-biloba e amido hidroxietílico hipertônico na atenuação de alterações decorrentes da isquemia e reperfusão de órgãos esplâncnicos em ratos. Não houve diferença estatística entre os grupos utilizando os fármacos e o grupo controle.

Outros métodos de avaliação da injúria tecidual têm sido utilizados em estudos experimentais, além da análise histológica. O principal objetivo é encontrar uma forma pouco invasiva de auxiliar no diagnóstico precoce da síndrome de isquemia e reperfusão mesentérica.

Em nosso meio, BRITO, ARAÚJO, ACÁCIO, ACÁCIO e REIS ${ }^{25}$ compararam a avaliação histológica e o estudo colorimétrico usando sal tetrazólico em ratos submetidos a isquemia e reperfusão mesentérica, mostrando haver equivalência quanto à sensibilidade dos métodos. Entretanto, o método não-histológico mais utilizado é a dosagem dos níveis teciduais e plasmáticos do malondialdeído, um radical lipídico que se encontra aumentado durante a reperfusão.

LO, CHEN, CHEN et al. ${ }^{28}$ relataram que a aminoguanidina foi capaz de reduzir os níveis de peroxidação lipídica intestinal e hepática, realizando-se isquemia ileal por oclusão da artéria mesentérica superior durante trinta minutos e reperfusão também por trinta minutos. Os níveis intestinais e pulmonares de malondialdeído foram menores no grupo em que se utilizou aminoguanidina que no grupo controle.

FERRER, ARICETA, GUERRERO et al. ${ }^{29}$ realizaram isquemia intestinal por sessenta minutos, seguida de cento e cinqüenta minutos de reperfusão, e utilizaram alopurinol e $\mathrm{N}$-acetilcisteína como protetores da injúria tecidual, obtendo $60 \%$ de redução da necrose intestinal medida em centímetros em comparação ao grupo controle. Entretanto, os demais dados analisados na avaliação da prevenção de lesão de reperfusão foram bastante insatisfatórios. O malondialdeído plasmático e o glutatião reduzido tiveram níveis aumentados nos tecidos intestinais, pulmonares e cardíacos avaliados. Além disso, não há relatos na literatura, a não ser um outro estudo dos mesmos autores ${ }^{30}$, deste método de avaliação de prevenção de lesão tecidual em que se mede em centímetros a área que sofreu necrose.

FERRER, ARICETA, GUERRERO et al. ${ }^{30}$ estudaram os efeitos da somatostatina na isquemia e reperfusão em ratos por oclusão da artéria mesentérica superior e observaram não haver melhora em relação ao grupo controle quanto aos parâmetros analisados: necrose em centímetros, amilase plasmática, malondialdeído plasmático e mieloperoxidase tecidual. A análise foi feita no intestino, pulmão e coração.

Outro estudo utilizando somatostatina como citoprotetor foi realizado por ARICETA, FERRER, GUERRERO, TELLECHEA, BALÉN e LERA ${ }^{23}$, no qual ratos foram submetidos a 60 minutos de isquemia por oclusão da artéria mesentérica superior seguidos por 90 minutos de reperfusão, demonstrando-se não haver sinais de peroxidação lipídica. Porém, houve um aumento importante dos níveis teciduais de mieloperoxidase, e, portanto, não eliminando a formação de radicais livres. Seria importante que novos trabalhos fossem realizados com esta substância para maiores esclarecimentos. 
KUMAMOTO, MATSUDA, KATAOBA e KOKUBA ${ }^{31}$ utilizaram aminosalicilatos administrados por via intestinal, realizando isquemia mesentérica por 10 minutos e reperfusão por 60 minutos. Utilizaram como método de avaliação a dosagem de substâncias tiobarbitúrico-ácido-reativas que, segundo os autores, são capazes de demonstrar o índice de peroxidação lipídica na mucosa intestinal de ratos. Os resultados mostraram um efeito positivo na prevenção de lesão de isquemia e reperfusão, porém, há que se levar em consideração o reduzido tempo de isquemia, período este que, segundo BOROS et al. ${ }^{24}$, não é suficiente para provocar lesões importantes. Além disso, com um reduzido tempo de isquemia, esperar-se-ia uma menor produção de hipoxantina e xantina-oxidase, que desencadeariam o processo de formação dos radicais livres em presença de oxigênio na fase de reperfusão.

YAMADA, MURASE, MAEDA et al. ${ }^{32}$ estudaram os efeitos do FR167653 em transplante ortotópico de intestino delgado em ratos. $\mathrm{O}$ enxerto foi mantido por doze horas em solução de Ringer lactato a $4{ }^{\circ} \mathrm{C}$. De quatorze animais estudados em cada grupo, sobreviveram por 48 horas apenas 11 ratos do grupo FR167653 e cinco do grupo controle. Os níveis plasmáticos de fator de necrose tumoral e interleucina-1 após o transplante foram menores que no grupo controle, sendo estas as principais substâncias a serem analisadas, segundo os autores, visto serem agentes pró-inflamatórios. Os níveis pulmonares de mieloperoxidase também estiveram diminuídos após 12 horas de reperfusão em relação ao grupo controle.

HAMMERMAN, GOLDSCMIDT, CAPLAN

et al. ${ }^{33} \mathrm{em}$ estudo experimental em ratos, analisaram os efeitos da pentoxifilina como mediadora da inibição da xantina-oxidase na isquemia mesentérica, realizando oclusão da artéria mesentérica superior por 45 minutos e reperfusão de 30 e 60 minutos, observando níveis significativamente maiores de xantina-oxidase e de xantina-desidrogenase no grupo controle em relação aos grupos utilizando a pentoxifilina. Entretanto, tais substâncias encontram-se aumentadas na fase de isquemia, e, embora seja provável que desencadeiem reações que poderiam levar à injúria tecidual, não são marcadores de lesão de reperfusão.

SILERI, BROWN, MORINI, RASTELLINI, BENEDETTI e CICALESE ${ }^{34}$ submeteram ratos a três episódios de isquemia por oclusão da artéria mesentérica superior durante 45 minutos a cada sete dias. Após quatro semanas do último procedimento de oclusão arterial analisaram o grupo controle e o grupo recebendo piruvato na dieta, concluindo que tal substância previne a injúria tecidual na isquemia e reperfusão intestinal, uma vez que a absorção de Dxilose no grupo estudado foi maior que a do grupo controle. Entretanto, segundo SHAN et al. ${ }^{35}$, com este período de isquemia, a regeneração das vilosidades intestinais ocorre por completo após 48 horas, mesmo sem qualquer tratamento.

KLOTZ et al. ${ }^{36}$ realizaram estudo prospectivo não randomizado em pacientes em pós-operatório de cirurgias cardíacas (revascularização miocárdica, troca de válvula aórtica e transplante cardíaco) com quadro clínico compatível com isquemia mesentérica. Submeteram 20 pacientes à arteriografia mesentérica, dos quais 14 tiveram o diagnóstico de isquemia mesentérica não-oclusiva. Estes 14 pacientes receberam papaverina por cateter posicionado na origem da artéria mesentérica superior. Nove tiveram melhora e cinco necessitaram tratamento operatório com ressecção intestinal segmentar. Destes cinco, três foram a óbito. A papaverina é um vasodilatador potente já utilizado em humanos sem efeitos colaterais ou complicações, segundo estes autores. Entretanto, cinco pacientes ( $35 \%$ ) não tiveram boa resposta com seu emprego e necessitaram intervenção cirúrgica. Também CALEB ${ }^{37}$ utilizou a papaverina por cateter posicionado na origem da artéria mesentérica superior em três pacientes com isquemia mesentérica após cirurgias cardíacas com circulação extracorpórea, porém, todos os pacientes foram a óbito. EKER, MALZAC, TEBOUL e JOURDAN ${ }^{38}$ relataram um caso de paciente com boa resposta à papaverina em isquemia mesentérica não-oclusiva após revascularização miocárdica com circulação extracorpórea.

Assim, pudemos observar que inúmeras substâncias foram avaliadas em suas capacidades de minimizar ou evitar os efeitos lesivos do processo de isquemia e reperfusão mesentérica, a maioria sem boa resposta, porém, algumas com efeitos promissores. Portanto, novos estudos são necessários a fim de se encontrar uma ou mais substâncias que possam ser empregadas nas situações de isquemia e reperfusão mesentérica em humanos, para que sejam evitados tratamentos de maior morbi-mortalidade como os atualmente utilizados. 
SUMMARY: A short time ago, it was believed that all of the organic alterations in the mesenteric ischemia were due to the total or partial obstruction of the intestinal arterial flow. As for the physiopathology of the ischemia process and mesenteric reperfusion, recent discoveries demonstrated that the free radicals, mainly, act during the reperfusion taking to the lesion much more important tecidual than the lesions that happen in the ischemia phase separately. So a new therapeutic possibility appears besides the surgical treatment when a certain substance could act in the way to inhibit or to minimize the cascade of alterations at the cellular level that culminate to the cellular lesion and death. We accomplished a wide revision of the current medical literature for the databases LILACS and MEDLINE looking to verify the studied drugs for this gol and the results obtained in the researches. We verified that countless substances have been appraised in experimental studies and most of them didn't show satisfactory results for their acceptance in practice clinic. Although some presented promising results, new studies are still needed to discover a substance that can be used in ischemia situations and mesenteric reperfusion in human beings to avoid interventionist treatments with high mortality rate.

Key words: Ischemia, reperfusion, experimental surgery

\section{REFERÊNCIAS BIBLIOGRÁFICAS}

1. Scannel G, Waxman K, Vaziri ND, Zhang J, Kaupke CJ, Jalali M, Hecht CC. Hypoxia-induced alterations of neutrophil membrane receptors. J Surg Res 1995; 59:141-5.

2. Pinheiro BV, Holanda MA, Araújo FG, Romaldini H. Lesão pulmonar de reperfusão. J Pneumol 1999; 25(2):124-36.

3. Meyer TN, Silva AL. Resposta celular ao estresse. Rev Assoc Med Bras 1999; 45(2): 181-8.

4. Parks DA, Granger DN. Contributions of ischemia and reperfusion to mucosal lesion formation. Am J Physiol 1986; 250: 749-53.

5. Marcuson RW. Ischaemic colitis. Clin Gatroenterol 1972; 1(3): 745-63.

6. Marston A. Acute intestinal ischaemia - Resection rather than revascularization. BMJ 1990; 301: 1174-5.

7. Reis Neto JA, Ribeiro AP. Trombose mesentérica inferior. Rev Ass Med Brasil 1973; 20(5): 189-90.

8. Kaminski DL, Keltner RM, Willman VL. Ischemic colitis. Arch Surg 1973; 106: 558-63.

9. Marston A, Pheils MT, Thomas L, Morson BC. Ischaemic colitis. Gut 1966; 7: 1-15.

10. Keighley MRB - Isquemia do colo e colite isquêmica - In: Keighley MRB, Williams NS (Ed) Cirurgia do Ânus, Reto e Colo, São Paulo-SP, Manole, 1998: 1846-66.

11. Yongming Y, Yan Y, Ye W, Zhiguo S, Zhiyong S. The role of gut as a cytokine-generating organ in remote organ dysfunction after intestinal ischemia and reperfusion. Chin Med J 1998; 111(6): 514-8.

12. Procianoy RS, Silveira RC. Síndrome hipóxico-isquêmica. J Pediatr 2001; 77(1): 63-70.

13. Anadol AZ, Bayram O, Dursun A, Ercan S. Role of endogenous endothelin peptides in intestinal ischaemia-reperfusion injury in rats. Prostaglandins Leukot Essent Fatty Acids 1998; 59(4): 279-83.
14. Santos CHM, Gomes OM, Pontes JCDV et al. Use of propofol (2,6 diisopropilphenol) as inhibitor of tissue lesion in mesenteric ischemia and reperfusion: experimental study in rats. Acta Cir Bras 2003, 18: 347-54.

15. Chiu CJ, McArdle AH, Brown R, Scott HJ, Gurd FN. Intestinal Mucosal Lesion in Low-Flow States. Arch Surg 1970; 101: 478-83.

16. Lee MA, McCauley RD, Kong SE, Hall JC. Pretreatment with glycine reduces the severity of warm intestinal ischemicreperfusion injury in the rat. Ann Plastic Surg 2001; 46(3): 320-6.

17. Macarenco RSS, Takahagi RU, Bardella LC, Sequeira JL, Yoshida WB. Estudo da ação do extrato de Gingko biloba e amido hidroxietílico hipertônico na atenuação de alterações decorrentes de isquemia e reperfusão de órgãos esplâncnicos em ratos. Acta Cir Bras jul/ago/set 2001; 16(3): 00-00. ISSN 0102-8650.

18. Biondo-Simões MLP, Greca FH, Ioshi S, Tawil IIE, Menini CM, Rampazzo JC. The influence of antibiotics on intestinal ischemia and reperfusion: experimental study in rats. Acta Cir Bras 2000; 15(3): 83-7.

19. Guo WH, Chan KL, Fung PPCW, Chan KW, Tam PKH. Nitric Oxide Protects Segmental Intestinal Grafts From Ischemia and Reperfusion Injury. Transpl Proc 2000; 32: 1297-8.

20. Ward DT, Lawson AS, Gallagher CM, Conner WC, SheaDonohue T. Sustained Nitric Oxide Production via L-Arginine Administration Ameliorates Effects of Intestinal IschemiaReperfusion. J Surg Res 2000; 89: 13-9.

21. Onen A, Deveci E, Inaloz SS, Isik B, Kilinc M. Histopathological assesment of the prophylactic effect of gingko-biloba extract on intestinal ischemia-reperfusion injury. Acta Gastroenterol Belg 1999; 62: 386-9.

22. Kaçmaz M, Ozturk HS, Karaayvaz M, Guven C, Durak I. Enzymatic antioxidant defence mechanism in rat intestinal tissue is changed after ischemia-reperfusion. Effects of an al- 
lopurinol plus antioxidant combination. Can J Surg 1999; 42: 427-31.

23. Ariceta J, Ferrer JV, Gerrero D, Tellechea E, Balén E, Lera JM. Metabolism of the Intestine With Intermediate Ischemia After Intestinal Ischemia-Reperfusion Injury: Therapeutic effects of Somatostatin. Transpl Proc 1999; 31: 2572.

24. Boros M, Takaichi S, Hatanaka K. Ischemia Time Dependency of Reperfusion Injury Following Complete Arterial Occlusion of the Rat Small Intestine. Transpl Proc 1995; 27(5): 2789-90.

25. Brito MVH, Araújo M, Acácio GJS, Acácio GJS, Reis JMC. Lesão Intestinal após Isquemia-Reperfusão: Estudo Comparativo usando Sal Tetrazólico (MTT) e Histologia. Acta Cir Bras jan/fev/mar 2001; 16(1): 00-00. ISSN 0102-8650.

26. Khanna A, Rossman JE, Fung HL, Caty MG. Attenuated Nitric Oxide Synthase Activity and Protein Expression Accompany Intestinal Ischemia/Reperfusion Injury in Rats. Biochem Biophys Res Commun 2000; 269: 160-4.

27. Durakbasa ÇU, Dagli TE, Mouni H, Haklar G, Bilsel AS, Yuksel M, Aktan AO. Nitric oxide and endothelin relationship in intestinal ischemia/reperfusion injury. Prostaglandins Leukot Essent Fatty Acids 1998; 59(6): 379-83.

28. Lo CC, Chen JC, Chen HM, Shyr MH, Lau YT, Lin JN, Chen MF. Aminoguanidine Attenuates Hemodynamic and Microcirculatory Derangement in Rat Intestinal Ischemia and Reperfusion. J Trauma 1999; 47(6): 1108-13.

29. Ferrer JV, Ariceta J, Guerrero D, Gomis T, Larrea MM, Balén E, Lera JM. Allopurinol and N-Acetylcysteine Avoid $60 \%$ of Intestinal Necrosis in an Ischemia-Reperfusion Experimental Model. Transpl Proc 1998; 30: 2672.

30. Studer W, Wu X. Supraceliac aortic cross-clamping and declamping. Effects of dopexamina and dopamine on systemic and mesenteric hemodynamics, metabolism and intestinal tonometry in a rat model. Acta Anaesthesiol Scand 2000; 44: 241-8.

31. Kumamoto T, Matsuda A, Kataoba M, Kokuba Y. Inhibition by Aminosalicylates of Lipid Peroxidation in Large Intestinal Mucosa after Mesenteric Ischemia/Reperfusion in the Rat. Jpn J Pharmacol 1997; 75:187-9.
32. Yamada T, Murase N, Maeda T, Ye Q, Sakamoto T, Terakura M, Starzi Te, Todo S. Protective Effect of TNF-alfa and IL-1 BETA Inhibitor FR167653 on Ischemia-Reperfusion Injury in Rat Small Intestinal Transplantation. Transpl Proc 1998; 30: 2638 .

33. Hammerman C, Goldschmidt D, Caplan MS, Kaplan M, Schimmel MS, Eidelman AI, Branski D, Hochman A. Amelioration of Ischemia-Reperfusion Injury in Rat Intestine by Pentoxifylline-Mediated Inhbition of Xanthine Oxidase. J Pediatr Gastroenterol Nutr 1999; 29: 69-74.

34. Sileri P, Brown M, Morini S, Rastellini C, Benedetti E, Cicalese L. Pyruvate prevents intestinal functional changes following ischemia-reperfusion injury. Transpl Proc 2001; 33: 852.

35. Shan Z, Baixiang J, Caty MG, Tjota A. Mucosa cell regeneration following intestinal ischemia/reperfusion injury in rats. Chin Med J 1997; 110(5): 338-40.

36. Klotz S, Vestring T, Rotker J, Schmidt C, Scheld HH, Schmid C. Diagnosis and Treatment of Nonocclusive Mesenteric Ischaemia After Open Heart Surgery. Ann Thorac Surg 2001; 72(5): 1583-6.

37. Caleb MG. Acute Bowel Ischemia after Coronary Bypass Surgery - A Catastrophic Event. Singapore Med J 2001; 12(1): 33-7.

38. Eker A, Malzac B, Teboul J, Jourdan J. Mesenteric ischemia after coronary artery bypass grafting: should local continuous intra-arterial perfusion with papaverine be regarded as a treatment?. Eur J Cardio Thorac Surg 1999; 15: 218-20.

Endereço para correspondência: CARLOS HENRIQUE MARQUES DOS SANTOS

Rua Aluísio de Azevedo, 606 - Jardim São Bento 79.004-050 - Campo Grande (MS)

E-mail: chmarques@terra.com.br 